

\section{Shirkah}

\section{Journal of Economics and Business \\ Vol. 1, No. 1, January-April 2016 \\ ISSN: 2503-4235 (p); 2503-4243 (e)}

\section{Editor in Chief}

Sri Walyoto

\section{Editorial Boards}

Ahmad Ihwan Setiawan, Faculty of Economics, Universitas Sebelas Maret

Dwi Condro Triono, Faculty of Islamic Economics and Business, IAIN

Surakarta

Fahmy Radhi, Faculty of Economics, Universitas Gadjah Mada

Jaka Sriyana, Faculty of Economics, Universitas Islam Indonesia

\section{Managing Editors}

M. Endy Saputro

M. Zainal Anwar

\section{Assistant to Editor}

Supriyanto

Shirkah Journal of Economics and Business is a peer-reviewed journal published three times a year (January-April, May-August and September-December) by Faculty of Islamic Economics and Business, Institut Agama Islam Negeri (IAIN/ State Institute for Islamic Studies) Surakarta Central Java, Indonesia. The main objective of Shirkah is to offer an academic space of exchange ideas and initiate the increase number of qualified article produced by postgraduate students, practitioners and academicians.

\section{Editorial Office}

Ruang Jurnal Shirkah

Lantai Dasar, Sayap Barat, Fakultas Ekonomi dan Bisnis Islam, IAIN Surakarta

Jln. Pandawa No. 1, Kartasura, Sukoharjo, Jawa Tengah Kode Pos. 57168

Phone (+62271) 781516 Fax: (+62271)782336

E-mail: shirkahiainsurakarta@gmail.com

Website: http://shirkah.or.id/ 


\section{Shirkah}

Journal of Economics and Business

Vol. 1, No. 1, January-April 2016

ISSN: 2503-4235 (p); 2503-4243 (e)

\section{Table of Contents}

\section{Articles}

Muthmainah

The Growth of Sharia Insurance in Indonesia 2015 - 2016

An Academic Forecast Analysis

Meilana Widyaningsih

$23-46$

The Effect of Material, Social and Activities Compensations

toward Work Performance Using Organizational Commitment

as The Mediator

Tri Wabyuni

$47-72$

The Influence of Technology Acceptance Model (TAM)

on The Users' Behavior of Sikesya Application in IAIN Surakarta

M. Nidaussalam

Mudaraba as a Model of Islamic Finance

Sri Maryati

$93-112$

Evaluating The Performance of Inventory Management

The Production Division of PT. Tiga Serangkai Surakarta

as a Case Study

Mardhiyaturrositaningsih

$113-126$

Sharia Banking's Profit Loss Finance in the Context of ASEAN Economic Community 


\title{
The Influence of Technology Acceptance Model (TAM) on The Users' Behavior of Sikesya Application in IAIN Surakarta
}

\author{
Tri Wahyuni \\ PT. Sukses Abadi Karya Inti - Sragen
}

\begin{abstract}
This study aimed at evaluating the student acceptance of SIKESYA (Sharia Financial System/Sikesya) application as the users by using the framework of Technology Acceptance Model (TAM) and its development. The constructs being tested in this research are perceived usefulness, perceived ease of use, experience, social influence, attitute toward behavior, behavioral intention, facilitating condition, and user behaviors. As much as 80 students has been chosen as sample which were determined using purposive sampling method. The data gathered was then analyzed using partial least square (PLS). The result showed that experience did not influence the perceived ease of use, on the other hand perceived usefulness has a positif influence toward users attitude and behavior in using Sikesya, while the perceived ease of use did not influence the users atttitude and behavior at all, since the students would still use it as it is an application used as part of university services. The attitude and behavior did not influence the behavioral intention, whereas the social influence has a positif effect on behavioral intention, yet the behavioral intention gave positif impact to user's behavior. On the other hand, facilitating condition has no effect toward users' behavior.
\end{abstract}

\section{Keywords:}

Sharia Financial System (SIKESYA), Technology Acceptance Model (TAM), IAIN Surakarta

Information system has started to apply on every organization, means every information system's learning and training is really needed by students. Practical class of Sistem Keuangan Syariah (SIKESYA) for 
Akuntansi Sharia student is very salient providing experience for the students therefore they will know information system (IT) to support their work. Some students still find difficulties in applying SIKESYA even though they have practiced it in class. These difficulties are due to SIKESYA system complexity and difficult pattern. The application and understanding's difficulty in SIKESYA application will badly affect SIKESYA application with system rejection and it will be failing system application.

Student's difficulty in system application is the system application problem as stated by Widiatmaka and Sensue (2014), "New system application will always create reaction to its users." The reaction is in acceptance or rejection. Davis (1993) said that low user's acceptance will affect new information system application's success. Davis (1993) said information system use's success depends on system user's acceptance. Low user's acceptance will affect system application success. The organization needs to know its manager and employers' appreciation on the use of information system. The organization's research on constructions will define manager and employers' acceptance on in use information system.

Students as the user of information system are also needed to be evaluated. The new system application such as SIKESYA not necessarily benefitted the students. It dues the accountancy process in the system does not provide help for the student, in fact it will bring difficulties. Therefore, this research is salient to evaluate applied system for accountancy students in money management. The model in use to research students' behavior is TAM. Technology Acceptance Model (TAM) is a new model with utilization and trusted convenience in behavior which will user practically ease-ready to use. Many information system user behavior's researches use TAM (Kusumo, 2010). TAM, theoretically, is the most correct model to explain how users accept a system (Handayani, Kustini, and Sunyoto, 2013). 
Technology Acceptance Model (TAM) is the correct method to predict user's intention in using the system, providing valuable information, and simple and easily applicable model. Many researchers stated TAM model is better than other models (Jogiyanto, 2007, p. 120). There are five main constructions on first Technology Acceptance Model's before modification, namely: perception utility, easiness perception utility, attitude on behavior, behavior's intention, and behavior (Jogiyanto, 2007, pp. 113-114). There are many researchers developing TAM theories to make it more useful.

Yusman (2013) used Technology Acceptance Model to study SIKD user's behavior. The tested construction in this research is utility perception construction, convenience perception, technology using attitude, technology using behavior intention, and real technology using. The research concludes TAM constructions truly underlie an individual's fact using. Handayani, Kusrini, and Sunyoto (2013) used TAM framework to test the affected factors of technology acceptance level. The TAM developing in this research adds usability construction. There are 3 TAM constructions in use, namely user perception on convenience, user perception on utility, and user attitude on technology using.

Rizal (2014) used TAM model to analyze project management information system acceptance. The tested variables for this purpose are usability convenience, utility, intention to use, likeness to use, and the real utility. Kusumo (2010) wanted to discern m-banking user acceptance with TAM framework using. Kusumo also added complexity variable to be tested in TAM framework. The research result shows system's complexity considered will enhance work performance.

Ari (2013) developed TAM with adding social effect construction and facilitator condition among 5 other constructions. Those 5 constructions are utility perception, utility convenience perception, attitude behavior, 
behavior's interest, and utility behavior. Retnoningrum and Jalil (2013) developed TAM with adding external variable namely user's experience and pleasure perception variable. This test shows individual experience in using technology will make technology using interaction easier, more fun, and satisfying. However the experience does not affect utility perception.

The research will retest constructions used by Ari (2013) with title "Technology Acceptance Model Effect and Its Development in Core Banking System Using Attitude." The researcher develops the current study with different object research and adding one tested construction. The previous study is on general bank at Malang Raya and next research is on SIKESYA at IAIN Surakarta. The adding construction is experience. Experience construction is also added on study by Retnoningrum and Jalil in TAM development. The researcher is also adding experience construction due to theoretically direct experience with information system will be intermediary direct relation of utility's purpose and convenience. Easy direct experience will support attitude in information system technology using.

\section{Utility Perception and Attitude Behavior: A Hypothesis}

The tested constructions in this research comes from Ari's (2013) research, namely utility perception, utility convenience perception, attitude behavior, social effect, attitude interest, and facilitator conditions. The researcher added experience construction to develop the study. Experience is one of the control variables used by Venkatesh et al. (2003) to test real attitude's interest and utility on technology. Therefore the researcher has hypothesis are:

$\mathrm{H}_{1}$ : experience affects utility convenience perception.

$\mathrm{H}_{2}$ : utility perception affects the SIKESYA using attitude behavior.

$\mathrm{H}_{3}$ : utility convenience perception affects SIKESYA using attitude behavior. 
$\mathrm{H}_{4}$ : attitude behavior affects the SIKESYA using attitude interest.

$\mathrm{H}_{5}$ : social effect affects the SIKESYA using attitude interest.

$\mathrm{H}_{6}$ : attitude interest affects the SIKESYA using behavior.

$\mathrm{H}_{7}:$ Facilitators' conditions affect the SIKESYA using attitude.

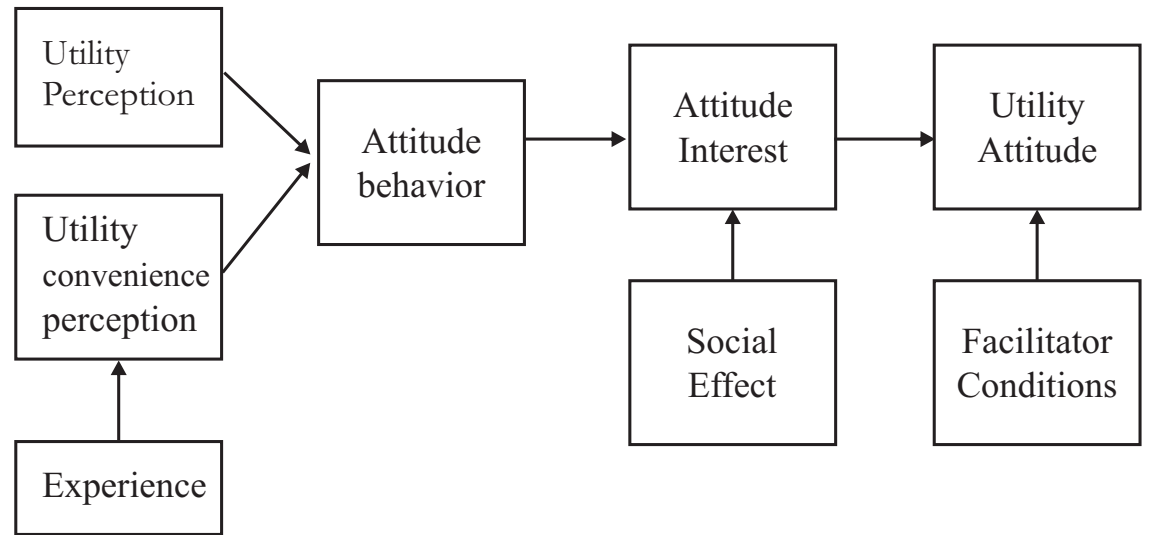

The population of this research is all students of Akuntansi Syariah Fakultas Ekonomi dan Bisnis Islam IAIN Surakarta students who take class of SIKESYA practice. The data are analyzed with multivariate. Total sample members are minimum $10 \mathrm{x}$ of studied variable, namely $10 \mathrm{X} 8=80$. It means the minimum samples of this research are 80 (Roscoe, as quoted in Sugiono, 2012, p. 133). The technique sampling in use is purposive sampling.

Data was intentionally gathered from questionnaire, interview, and bibliographical study. The questionnaire purpose contains students' response on the researchers' question. Interview's purpose is to grasp information on studied issues, and bibliographic study is supporting research data with sources from books, journal under graduate thesis, and graduate thesis. The concept in this research is Technology Acceptance Model (TAM) and its development by Ari (2013). It is tested with 87 variables/ constructions, namely 4 Exogenous latent Variables and 4 Endogenous Latent Variables. 
The exogenous latent variables are:
a. Utility perception;
b. Experience;
c. Social effect;
d. Facilitator conditions.

The endogenous latent variables are:

a. Utility convenience perception;

b. Behavior attitude;

c. Behavior interest;

d. User behavior.

The instrument in use to collect data is questionnaire sheet. The measurement design scale in use is Likert's scale with score 1 to 5:

Construction, definition, and indicators used in the research are explained on table below:

\section{Variable Operational Definition}

\begin{tabular}{|l|l|l|l|}
\hline \multicolumn{1}{|c|}{ Source } & Construction & \multicolumn{1}{|c|}{ Definition } & \multicolumn{1}{|c|}{ Indicators } \\
\hline Ari (2013) & Utility perception & $\begin{array}{l}\text { Individual's assurance } \\
\text { in using SIKESYA } \\
\text { to enhance his/ her } \\
\text { performance }\end{array}$ & $\begin{array}{l}\text { 1. Finish the job faster } \\
\text { 2. Performance enhancement } \\
\text { 3. Productivity enhancement } \\
\text { 4. Effectiveness enhancement } \\
\text { 5. Made the job easier } \\
\text { 6. Useful in job }\end{array}$ \\
\hline $\begin{array}{l}\text { J o g i y a n t o } \\
\text { (2007) }\end{array}$ & Experience & $\begin{array}{l}\text { Learning process and } \\
\text { good behavioral potential } \\
\text { development's addition } \\
\text { in formal and non- } \\
\text { formal, or a process to } \\
\text { bring a person into higher } \\
\text { behavior. }\end{array}$ & $\begin{array}{l}\text { 1. Followed training } \\
\text { Gaining experience in } \\
\text { system application }\end{array}$ \\
\hline
\end{tabular}




\begin{tabular}{|c|c|c|c|}
\hline Ari (2013) & Social effect & $\begin{array}{lr}\text { Individual's } & \text { assurance } \\
\text { on interest perception, } \\
\text { believed by other } \\
\text { individuals will affect } \\
\text { application use. }\end{array}$ & $\begin{array}{l}\text { 1. Other individual effect } \\
\text { 2. Important individual effect } \\
\text { 3. Senior management effect } \\
\text { 4. Organization effect }\end{array}$ \\
\hline Ari (2013) & $\begin{array}{l}\text { F a cilit a t o r } \\
\text { Condition }\end{array}$ & $\begin{array}{l}\text { Individual's assurance } \\
\text { on organization and } \\
\text { technical infrastructure } \\
\text { available to support } \\
\text { information system in use }\end{array}$ & $\begin{array}{l}\text { 1. Resources } \\
\text { 2. Knowledge } \\
\text { 3. Compatibility } \\
\text { 4. Certain individual or } \\
\text { group contribution }\end{array}$ \\
\hline Ari (2013) & $\begin{array}{l}\mathrm{U} \text { t i l i t y } \\
\text { Conven i ence } \\
\text { Perception }\end{array}$ & $\begin{array}{l}\text { Individual's assurance } \\
\text { SIKESYA r using } \\
\text { application will be free of } \\
\text { effort }\end{array}$ & $\begin{array}{l}\text { 1. Easy to learn } \\
\text { 2. Easy to arrange } \\
\text { 3. Clear and understandable } \\
\text { 4. Easy to be skillful } \\
\text { 5. Flexible } \\
\text { 6. Easy to use }\end{array}$ \\
\hline Ari (2013) & Behavior Attitude & $\begin{array}{l}\text { Individual like and } \\
\text { dislike feeling of new } \\
\text { information system he/ } \\
\text { she is using }\end{array}$ & $\begin{array}{l}\text { 1. Good or bad feeling } \\
\text { 2. Like or dislike feeling } \\
\text { 3. Profit or loss feeling } \\
\text { 4. Interest or disinterest } \\
\text { feeling }\end{array}$ \\
\hline Ari (2013) & Behavior Interest & $\begin{array}{l}\text { Individual desire or } \\
\text { interest to do certain } \\
\text { action. An individual will } \\
\text { act base on desire to do it. }\end{array}$ & $\begin{array}{l}\text { 1. Intention to use } \\
\text { 2. Inclination to use } \\
\text { 3. Prediction to keep using in } \\
\text { the future } \\
\text { 4. Intention to use in the } \\
\text { future }\end{array}$ \\
\hline Ari (2013) & Utility behavior & $\begin{array}{l}\text { Individual real behavior } \\
\text { in using information } \\
\text { system }\end{array}$ & $\begin{array}{l}\text { 1. Utility period } \\
\text { 2. Utility Frequency }\end{array}$ \\
\hline
\end{tabular}

Data analysis technique in use is Partial Least Square (LPS) with SmartPLS 2.0 M3 application. PLS is a multivariate statistic technique, using comparison of double dependent variables and double independent variables. PLS measures model test and structural model test. Measurement model is use to test validity and reliability. Structural model is used for hypothesis test (Jogiyanto, 2009, pp. 11-14). 
Testing Results

Measurement model test is carried out with SmartPLS 2.0 M3 program. There are two main established parameters, namely construction validity test and validity test. Construction validity test consists of convergent validity test and discriminant validity test. There are 80 samples.

\section{Convergent validity test}

1) Utility Perception

Outer Loadings (Mean, STDEV, T-Values)

\begin{tabular}{|l|r|r|r|r|r|c|}
\hline & $\begin{array}{c}\text { Original } \\
\text { Sample } \\
(\mathrm{O})\end{array}$ & $\begin{array}{c}\text { Sample } \\
\text { Mean } \\
(\mathrm{M})\end{array}$ & $($ STDEV $)$ & $($ STERR $)$ & T Statistic & $\begin{array}{c}\text { Expla- } \\
\text { nation }\end{array}$ \\
\hline $\mathrm{KP} 1<-\mathrm{KP}$ & 0.819 & 0.810 & 0.057 & 0.057 & 14.302751 & Valid \\
\hline $\mathrm{KP} 2<-\mathrm{KP}$ & 0.821 & 0.822 & 0.037 & 0.037 & 22.370546 & Valid \\
\hline $\mathrm{KP} 3<-\mathrm{KP}$ & 0.838 & 0.817 & 0.071 & 0.071 & 11.803266 & Valid \\
\hline $\mathrm{KP} 4<-\mathrm{KP}$ & 0.765 & 0.742 & 0.106 & 0.106 & 7.216747 & Valid \\
\hline $\mathrm{KP} 5<-\mathrm{KP}$ & 0.525 & 0.537 & 0.122 & 0.122 & 4.299363 & Valid \\
\hline $\mathrm{KP} 6<-\mathrm{KP}$ & 0.617 & 0.599 & 0.121 & 0.121 & 5.108766 & Valid \\
\hline
\end{tabular}

Source: SmartPLS 2.0 M3 Processed Data Result, 2015

Base on the Outer Loadings table above, all indicators of utility construction perception has fulfilled convergent validity due to score higher than 0.5 .

2) Experience

Outer Loadings (Mean, STDEV, T-Values)

\begin{tabular}{|l|c|c|r|r|r|c|}
\hline & $\begin{array}{c}\text { Original } \\
\text { Sample } \\
(\mathrm{O})\end{array}$ & $\begin{array}{c}\text { Sample } \\
\text { Mean } \\
(\mathrm{M})\end{array}$ & (STDEV) & (STERR) & T Statistic & $\begin{array}{c}\text { Expla- } \\
\text { nation }\end{array}$ \\
\hline P1 <- P & 0.898 & 0.763 & 0.358 & 0.358 & 2.506377 & Valid \\
\hline P2 <- P & 0.774 & 0.609 & 0.459 & 0.459 & 1.688748 & Valid \\
\hline
\end{tabular}

Source: SmartPLS 2.0 M3 Processed Data Result, 2015 
Base on the Outer Loadings table above, all indicators of experience construction has fulfilled convergent validity due to score higher than 0.5 .

3) Social Effect

Outer Loadings (Mean, STDEV, T-Values)

\begin{tabular}{|l|c|c|r|r|r|c|}
\hline & $\begin{array}{c}\text { Original } \\
\text { Sample } \\
(\mathrm{O})\end{array}$ & $\begin{array}{c}\text { Sample } \\
\text { Mean } \\
(\mathrm{M})\end{array}$ & (STDEV) & (STERR) & T Statistic & $\begin{array}{c}\text { Explana- } \\
\text { tion }\end{array}$ \\
\hline PS1 <- PS & 0.763 & 0.772 & 0.082 & 0.082 & 9.279498 & Valid \\
\hline PS2 <- PS & 0.778 & 0.758 & 0.112 & 0.112 & 6.971746 & Valid \\
\hline PS3 <- PS & 0.733 & 0.696 & 0.158 & 0.158 & 4.632524 & Valid \\
\hline PS4 <- PS & 0.616 & 0.577 & 0.180 & 0.180 & 3.418354 & Valid \\
\hline
\end{tabular}

Source: SmartPLS 2.0 M3 Processed Data Result, 2015

Base on the Outer Loadings table above, all indicators of social effect construction has fulfilled convergent validity due to score higher than 0.5 .

4) Facilitator Conditions

Outer Loadings (Mean, STDEV, T-Values)

\begin{tabular}{|l|r|r|r|r|r|c|}
\hline & $\begin{array}{c}\text { Original } \\
\text { Sample } \\
(\mathrm{O})\end{array}$ & $\begin{array}{c}\text { Sample } \\
\text { Mean } \\
(\mathrm{M})\end{array}$ & (STDEV) & (STERR) & T Statistics & $\begin{array}{c}\text { Expla- } \\
\text { nation }\end{array}$ \\
\hline KKP1 <- KKP & 0.706 & 0.658 & 0.175 & 0.175 & 4.039167 & Valid \\
\hline KKP2 <- KKP & 0.497 & 0.454 & 0.240 & 0.240 & 2.073766 & $\begin{array}{c}\text { Not } \\
\text { valid }\end{array}$ \\
\hline KKP3 <- KKP & 0.754 & 0.679 & 0.218 & 0.218 & 3.463192 & Valid \\
\hline KKP4 <- KKP & 0.800 & 0.708 & 0.220 & 0.221 & 3.625847 & Valid \\
\hline KKP5 <- KKP & 0.112 & 0.166 & 0.284 & 0.285 & 0.393948 & $\begin{array}{c}\text { Not } \\
\text { valid }\end{array}$ \\
\hline
\end{tabular}

Source: SmartPLS 2.0 M3 Processed Data Result, 2015 
Base on the Outer Loadings table above, all indicators of KPP1, $\mathrm{KPP} 3$, and KPP4 has fulfilled convergent validity criteria due to loading scale score higher than 0.5. KPP2 and KPP5 have not fulfilled the validity; therefore these indicators will be erased and re-estimated. After reestimation the values shown on the table are:

Outer Loadings (Mean, STDEV, T-Values)

\begin{tabular}{|l|c|c|r|r|r|c|}
\hline & $\begin{array}{c}\text { Original } \\
\text { Sample } \\
(\mathrm{O})\end{array}$ & $\begin{array}{c}\text { Sample } \\
\text { Mean } \\
(\mathrm{M})\end{array}$ & (STDEV) & (STERR) & T Statistic & $\begin{array}{c}\text { Expla- } \\
\text { nation }\end{array}$ \\
\hline KKP1 <- KKP & 0.698 & 0.698 & 0.152 & 0.152 & 4.586533 & Valid \\
\hline KKP3 <- KKP & 0.812 & 0.761 & 0.197 & 0.197 & 4.122672 & Valid \\
\hline KKP4 <- KKP & 0.826 & 0.765 & 0.192 & 0.192 & 4.313475 & Valid \\
\hline
\end{tabular}

Source: SmartPLS 2.0 M3 Processed Data Result, 2015

The result above shows loading score values have fulfilled determined convergent validity standard. It means there are three facilitator conditions indicators which will be processed on the next level.

5) Utility Convenience Perception

Outer Loadings (Mean, STDEV, T-Values)

\begin{tabular}{|l|r|r|r|r|r|c|}
\hline & $\begin{array}{c}\text { Original } \\
\text { Sample } \\
(\mathrm{O})\end{array}$ & $\begin{array}{c}\text { Sample } \\
\text { Mean } \\
(\mathrm{M})\end{array}$ & $($ STDEV $)$ & (STERR) & T Statistics & $\begin{array}{c}\text { Expla- } \\
\text { nation }\end{array}$ \\
\hline KPP1 <- KPP & 0.633 & 0.552 & 0.218 & 0.218304 & 2.89898 & Valid \\
\hline KPP2 <- KPP & 0.741 & 0.700 & 0.141 & 0.141462 & 5.238559 & Valid \\
\hline KPP3 <- KPP & 0.771 & 0.734 & 0.136 & 0.135916 & 5.669334 & Valid \\
\hline KPP4 <- KPP & 0.646 & 0.628 & 0.182 & 0.182049 & 3.548145 & Valid \\
\hline KPP5 <- KPP & 0.656 & 0.644 & 0.159 & 0.158873 & 4.12635 & Valid \\
\hline KPP6 <- KPP & 0.720 & 0.693 & 0.145 & 0.144842 & 4.972123 & Valid \\
\hline KPP7 <- KPP & -0.487 & -0.476 & 0.158 & 0.157536 & 3.093741 & Not valid \\
\hline
\end{tabular}

Source: SmartPLS 2.0 M3 Processed Data Result, 2015 
Base on the Outer Load in table above, all indicators of utility convenience construction perception have fulfilled convergent validity due to score higher than 0.5 . There is one indicator has not fulfill validity namely KPP7. This indicator is erased and re-estimated. The value after re-estimation is shown on table below:

Outer Loadings (Mean, STDEV, T-Values)

\begin{tabular}{|l|r|r|r|r|r|c|}
\hline & $\begin{array}{c}\text { Original } \\
\text { Sample } \\
(\mathrm{O})\end{array}$ & $\begin{array}{c}\text { Sample } \\
\text { Mean } \\
(\mathrm{M})\end{array}$ & $($ STDEV $)$ & (STERR) & T Statistic & $\begin{array}{c}\text { Expla- } \\
\text { nation }\end{array}$ \\
\hline KPP1 <- KPP & 0.649 & 0.578 & 0.221 & 0.221 & 2.93192 & Valid \\
\hline KPP2 <- KPP & 0.750 & 0.715 & 0.120 & 0.120 & 6.276854 & Valid \\
\hline KPP3 <- KPP & 0.774 & 0.741 & 0.107 & 0.107 & 7.258674 & Valid \\
\hline KPP4 <- KPP & 0.639 & 0.627 & 0.160 & 0.160 & 4.00516 & Valid \\
\hline KPP5 <- KPP & 0.657 & 0.657 & 0.112 & 0.112 & 5.861532 & Valid \\
\hline KPP6 <- KPP & 0.712 & 0.691 & 0.133 & 0.133 & 5.370543 & Valid \\
\hline
\end{tabular}

Source: SmartPLS 2.0 M3 Processed Data Result, 2015

The result above shows loading score values of all indicators has fulfilled determined convergent validity standard. It means there are six indicators of utility convenience perception to process on the next level.

6) Behavior Attitude

Outer Loadings (Mean, STDEV, T-Values)

\begin{tabular}{|l|r|r|r|r|r|c|}
\hline & $\begin{array}{c}\text { Original } \\
\text { Sample } \\
(\mathrm{O})\end{array}$ & $\begin{array}{c}\text { Sample } \\
\text { Mean } \\
(\mathrm{M})\end{array}$ & (STDEV) & (STERR) & T Statistic & Keterangan \\
\hline SP1 <- SP & 0.842 & 0.828 & 0.061 & 0.061 & 13.766418 & Valid \\
\hline SP2 <- SP & 0.796 & 0.788 & 0.063 & 0.063 & 12.673008 & Valid \\
\hline SP3 <- SP & 0.673 & 0.660 & 0.127 & 0.127 & 5.312012 & Valid \\
\hline SP4 <- SP & 0.752 & 0.753 & 0.066 & 0.066 & 11.401654 & Valid \\
\hline
\end{tabular}

Source: SmartPLS 2.0 M3 Processed Data Result, 2015 
The result above shows all indicators loading score values of behavior attitude construction has fulfilled convergent validity due to score higher than 0.5 .

7) Behavior Interest

Outer Loadings (Mean, STDEV, T-Values)

\begin{tabular}{|l|r|r|r|r|r|c|}
\hline & $\begin{array}{c}\text { Original } \\
\text { Sample } \\
(\mathrm{O})\end{array}$ & $\begin{array}{c}\text { Sample } \\
\text { Mean } \\
(\mathrm{M})\end{array}$ & (STDEV) & (STERR) & T Statistic & $\begin{array}{c}\text { Expla- } \\
\text { nation }\end{array}$ \\
\hline MP1 <- MP & 0.827 & 0.816 & 0.061 & 0.061 & 13.452359 & Valid \\
\hline MP2 <- MP & 0.845 & 0.840 & 0.047 & 0.047 & 18.06518 & Valid \\
\hline MP3 <- MP & 0.900 & 0.889 & 0.032 & 0.032 & 27.776836 & Valid \\
\hline MP4 <- MP & 0.845 & 0.843 & 0.044 & 0.044 & 19.263172 & Valid \\
\hline
\end{tabular}

Source: SmartPLS 2.0 M3 Processed Data Result, 2015

The result above shows all indicators loading score values of behavior interest construction has fulfilled convergent validity due to loading score value higher than 0.5 .

8) Utility Behavior

Table 11

Outer Loadings (Mean, STDEV, T-Values)

\begin{tabular}{|l|c|c|r|r|r|c|}
\hline & $\begin{array}{c}\text { Original } \\
\text { Sample } \\
(\mathrm{O})\end{array}$ & $\begin{array}{c}\text { Sample } \\
\text { Mean } \\
(\mathrm{M})\end{array}$ & (STDEV) & (STERR) & T Statistic & $\begin{array}{c}\text { Expla- } \\
\text { nation }\end{array}$ \\
\hline $\mathrm{P} 1<-\mathrm{P}$ & 0.878 & 0.700 & 0.449 & 0.449 & 1.954255 & Valid \\
\hline $\mathrm{P} 2<-\mathrm{P}$ & 0.801 & 0.549 & 0.541 & 0.541 & 1.480286 & Valid \\
\hline
\end{tabular}

Source: SmartPLS 2.0 M3 Processed Data Result, 2015 
Base on the calculation above all indicators loading score values of user behavior construction has fulfilled convergent validity due to score loading value higher than 0.5 .

\section{Discriminant Validity Test}

Measured parameter in this test is comparing the root of AVE (table 4.17) of a construction should be higher than latent inter variable correlation (table 4.18), or watch cross loading.

Discriminant validity test result shows on the table below:

Average Variance Extracted (AVE)

\begin{tabular}{|c|c|c|}
\hline & AVE & AKAR AVE \\
\hline KKP & 0.515743 & 0.718152 \\
\hline KP & 0.548006 & 0.740274 \\
\hline KPP & 0.488425 & 0.698874 \\
\hline MP & 0.726429 & 0.852308 \\
\hline P & 0.703125 & 0.838525 \\
\hline PP & 0.788192 & 0.887802 \\
\hline PS & 0.526049 & 0.725292 \\
\hline SP & 0.590062 & 0.768155 \\
\hline
\end{tabular}

Source: SmartPLS 2.0 M3 Processed Data Result, 2015

Table 13

Latent Variable Correlation

\begin{tabular}{|l|r|r|l|l|l|l|l|l|}
\hline & KKP & KP & KPP & MP & P & PP & PS & SP \\
\hline KKP & 1 & & & & & & & \\
\hline KP & 0.392656 & 1 & & & & & & \\
\hline KPP & 0.509518 & 0.617952 & 1 & & & & & \\
\hline MP & 0.675152 & 0.440127 & 0.588526 & 1 & & & & \\
\hline P & 0.175615 & 0.405908 & 0.182253 & 0.155717 & 1 & & & \\
\hline PP & 0.358925 & 0.173792 & 0.266353 & 0.607217 & 0.141126 & 1 & & \\
\hline
\end{tabular}

Vol. 1 No. 1, January - April 2016 


\begin{tabular}{|l|r|r|r|r|r|r|r|r|}
\hline PS & 0.588547 & 0.5528 & 0.473174 & 0.587696 & 0.248413 & 0.397496 & 1 & \\
\hline SP & 0.455949 & 0.687461 & 0.52784 & 0.505548 & 0.341086 & 0.400042 & 0.669748 & 1 \\
\hline
\end{tabular}

Source: SmartPLS 2.0 M3 Processed Data Result, 2015

Base on the comparison of AVE root value on table 4.17 and inter latent variable correlation coefficient on the table above, concluded the indicator measurement of this research has consummated discriminant validity criteria.

\section{Reliability Test}

Measurement reliability shows a concept and variable measurement's instrument stability and consistency. Reliability is measured with observing the Cronbach's Alpha and Composite Reliability values. Cronbach's Alpha measures the real value of construction reliability. The reliable construction should have Cronbach's Alpha value > 0.6 and Composite Reliability should be $>0.7$. The construction reliability test result shows on the table below:

Cronbach's Alpha and Composite Reliability Value

\begin{tabular}{|c|c|c|}
\hline & $\begin{array}{c}\text { Cronbach's } \\
\text { Alpha }\end{array}$ & $\begin{array}{c}\text { Composite } \\
\text { Reliability }\end{array}$ \\
\hline KKP & 0.688777 & 0.806482 \\
\hline KP & 0.828049 & 0.87638 \\
\hline KPP & 0.792525 & 0.850669 \\
\hline MP & 0.874262 & 0.913892 \\
\hline P & 0.588778 & 0.824901 \\
\hline PP & 0.731522 & 0.881544 \\
\hline PS & 0.712506 & 0.815006 \\
\hline SP & 0.768617 & 0.851171 \\
\hline
\end{tabular}

Source: SmartPLS 2.0 M3 Processed Data Result, 2015

Vol. 1 No. 1, January - April 2016 
The table above shows most of constructions have Cronbach's Alpha value higher than 0.6 and Composite Reliability value higher than 0.7. There is one construction with Cronbach's Alpha value smaller than 0.6 , experience construction. This construction is considered reliable due to in term Composite Reliability value of 0.7 . The conclusion is all measurement in this research is reliable.

\section{Structural Model Test (Inner Model)}

\section{Goodness of Fit Model Test}

This test is carried out with R-Square value observation with goodness of fit model test. The test result is shown on the table below:

R-Square

\begin{tabular}{|c|c|}
\hline & R-Square \\
\hline KKP & \\
\hline KP & \\
\hline KPP & 0.033216 \\
\hline MP & 0.368114 \\
\hline P & \\
\hline PP & 0.372676 \\
\hline PS & \\
\hline SP & 0.489772 \\
\hline
\end{tabular}

Source: SmartPLS 2.0 M3 Processed Data Result, 2015

Table R-Square above shows the utility convenience construction perception is affected by $3 \%$ experience construction, behavior attitude construction is affected by $49 \%$ utility convenience construction perception, behavior interest construction is affected by $37 \%$ behavior attitude construction and social effect construction, and utility behavior construction is affected by $37 \%$ facilitator conditions and behavior 
intention construction.

2. Significant Test

Significant test is carried out with observing path coefficient value, namely coefficient showing significant level in hypothesis test. The hypothesis used in this research is one tail hypothesis. The hypothesis will be accepted whether it has t-statistic value higher than 1.64. Significant test results with SmartPLS 2.0 M3 program are:

\section{PLS Bootstrapping}

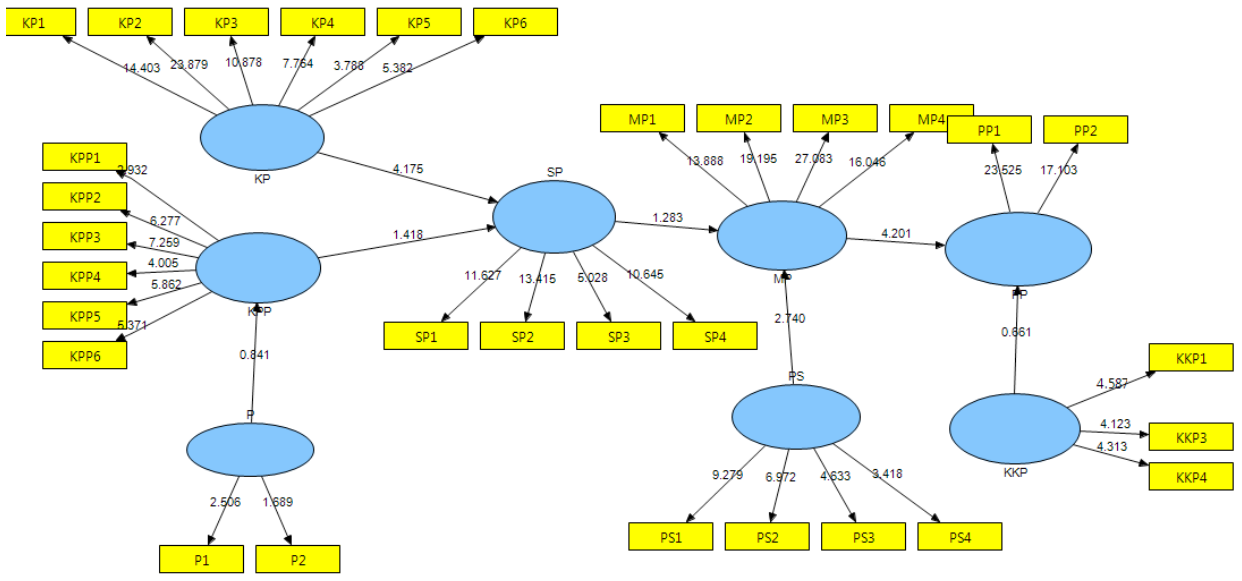

Source: SmartPLS 2.0 M3 Processed Data Result, 2015 
Path Coefficient Result

\begin{tabular}{|l|c|r|r|r|r|l|}
\hline & $\begin{array}{c}\text { Original } \\
\text { Sample } \\
(\mathrm{O})\end{array}$ & $\begin{array}{c}\text { Sample } \\
\text { Mean (M) }\end{array}$ & $\begin{array}{c}\text { Standard } \\
\text { Deviation } \\
(\text { STDEV) }\end{array}$ & $\begin{array}{c}\text { Standard } \\
\text { Error } \\
(\text { STERR })\end{array}$ & $\begin{array}{c}\mathrm{T} \\
\text { Statistics } \\
(\mid \mathrm{O} / \\
\text { STERR })\end{array}$ & Decision \\
\hline $\mathrm{P}>\mathrm{KPP}$ & 0.182253 & 0.202641 & 0.216821 & 0.216821 & 0.840569 & $\mathrm{H}_{1}$ rejected \\
\hline $\mathrm{KP}>\mathrm{SP}$ & 0.584469 & 0.555083 & 0.13998 & 0.13998 & 4.175385 & $\mathrm{H}_{2}$ accepted \\
\hline $\mathrm{KPP}>\mathrm{SP}$ & 0.166666 & 0.205362 & 0.117563 & 0.117563 & 1.417672 & $\mathrm{H}_{3}$ rejected \\
\hline $\mathrm{SP}>\mathrm{MP}$ & 0.202997 & 0.20539 & 0.158185 & 0.158185 & 1.283291 & $\mathrm{H}_{4}$ rejected \\
\hline $\mathrm{PS}>\mathrm{MP}$ & 0.451739 & 0.47777 & 0.164883 & 0.164883 & 2.739752 & $\mathrm{H}_{5}$ accepted \\
\hline $\mathrm{MP}>\mathrm{PP}$ & 0.670541 & 0.633781 & 0.159625 & 0.159625 & 4.200721 & $\mathrm{H}_{6}$ accepted \\
\hline $\mathrm{KKP}>\mathrm{PP}$ & -0.09379 & -0.04591 & 0.141873 & 0.141873 & 0.661103 & $\mathrm{H}_{7}$ rejected \\
\hline
\end{tabular}

Source: SmartPLS 2.0 M3 Processed Data Result, 2015

\section{Testing Result: An Academic Discussion}

$\mathrm{H}_{1}$ statistic test result is $\mathrm{t}_{\text {count }}=0.840569$ and beta coefficient value $=$ 0.182253 . It means $\mathrm{t}_{\text {count }}<\mathrm{t}_{\text {table }}$ and $\mathrm{H}_{1}$ is rejected. Experience construction does not have significant effect on utility convenience perception. It means an individual with experience or training does not provide him/ her with convenience in system or application using. It due to Akutansi Syariah students who join training is without intensive using. The training could not provide convenience in SIKESYA using.

$\mathrm{H}_{2}$ test result shows utility perception has positive effect on behavior attitude on SIKESYA. It shows on $\mathrm{t}_{\text {count }}$ value $4.175385>\mathrm{t}_{\text {table }}$ value 1.645 with $=0.05$. Beta coefficient value shows positive effect of 0.584469 . It shows the higher an individual trusts on SIKESYA using will enhance performance, therefore he/ she will like SIKESYA more. Students with positive attitude toward SIKESYA due to its provided utility value, therefore it will satisfy the application using. The research result is similar to those of Yusman (2013) and Ari (2013). 
$\mathrm{H}_{3}$ test result is $\mathrm{t}_{\text {count }}$ value of 1.417672 , therefore $\mathrm{H}_{3}$ statement is rejected. It proved utility convenience perception does not affect the behavior attitude in SIKESYA using. User in this research context is student. They use SIKESYA as obligation due to SIKESYA practice class. The students' easiness or difficulty will not affect their using. This research result is similar with Kartika's (2013).

$\mathrm{H}_{4}$ test result shows $t_{\text {count }}$ value of 1.283291 . It means $\mathrm{H}_{4}$ is rejected, and concludes behavior attitude does not have significant effect on behavior interest on SIKESYA using. It means an individual likeness on a system does not guarantee his/ her interest on the system. The students are using SIKESYA due to their interest not their likeness. Their interest is more on performance to support and demand on them to use the system. It is similar with the research of Taylor and Todd (1995) mentioned in Fathinah and Baridwan (2012).

Test on social effect and behavior interest shows $t_{\text {count }}$ value of 2.739752. It means $\mathrm{H}_{5}$ is accepted. Effect direction is positive, shown by the positive beta coefficient value, of 0.451739 . Therefore the higher the support of people around him/ her, the higher his/ her interest in SIKESYA using. Friend and organization's support will enhance student interest in using SIKESYA application. This result is similar to Ari's research (2013) and Handayani's (2005)

$\mathrm{H}_{6}$ test result shows beta coefficient value of 0.670541 , and $\mathrm{t}_{\text {count }}$ of 4.200721. Therefore $\mathrm{t}_{\text {count }}>\mathrm{t}_{\text {table }}$, means $\mathrm{H}_{6}$ is accepted. It means behavior interest has positive effect on individual behavior in SIKESYA using. Therefore the higher an individual behavior on SIKESYA using has a background of higher interest. This result is supporting the theory. It is similar with the research results of Ari (2013), Fathinah and Baridwan (2012), Davis (1989), and Handayani (2005). 
$\mathrm{H}_{7}$ is not accepted due to $\mathrm{t}_{\text {count }}$ of 0.661103 is less than $\mathrm{t}_{\text {table }}$. It means individual trust level on organization and technical infrastructure does not affect the individual behavior on SIKESYA using. Respondent inclines to agree on available organization and technical infrastructure in supporting SIKESYA. This research result shows no effect of facilitator conditions on utility behavior. Base on Ari research (2013) it dues to hygiene factor. This factor is a term to describe working aspect to avoid unsatisfied condition for an individual.

Hygiene factor is part of theory two factors. This theory said individual behavior is affected by two factors, namely motivation factor and hygiene factor. The research result shows there is no motivation of the user to use system due to likeness and self-motivation. It is due to demand on him/ her to use the system. This result is similar with Ari's research (2013).

\section{CONCLUSION}

The research purpose is finding the effect of Technology Acceptance Model (TAM) on user behavior in using Sistem Informasi Keuangan Syariah (SIKESYA) at IAIN Surakarta. Tested constructions in this research are experience, utility convenience perception, utility perception, behavior attitude, behavior interest, social effect, facilitator conditions, and user behavior. The conclusions are:

1. Experience does not affect the utility convenience perception dues to accountancy students' limited experience with courses in class and not on intensive activities.

2. Utility perception has positive effect on behavior attitude in SIKESYA using, therefore the higher SIKESYA utility the higher student appreciation on this system. 
3. Utility convenience perception does not affect behavior attitude in using SIKESYA. Students will still use SIKESYA without consideration on its difficulties or easiness.

4. Behavior attitude does not affect the behavior interest in using SIKESYA. Students use SIKESYA dues on demand to use it, not dues to likeness.

5. Social effect has positive effect in behavior attitude in using SIKESYA. Higher support from the people around will enhance student using SIKESYA.

6. Behavior interest has positive effect on SIKESYA utility behavior. The higher student interest, the higher potential of student in using SIKESYA.

7. Facilitator conditions do not affect SIKESYA utility behavior. It is due to facilitator conditions is a hygiene factor. It is a term to describe working aspect to avoid an individual dissatisfaction.

This research could not generalize due to its limited performed on students of Akuntansi Syariah Institut Program Agama Islam Negeri Surakarta. Tested variables in this research are experience, utility convenience perception, utility perception, behavior attitude, behavior interest, social effect, facilitator conditions, and user behavior. There are other variables able to affect SIKESYA using others than mentioned above. 1

SIKESYA application using in classes for institutions application indicates good utility of SIKESYA. Students' good behavior on SIKESYA is due to provided utility value. Even though the students gain experience of SIKESYA, it does not provide easiness, and it is not an easy application. It could be overcome with higher training and using proportions which will lessen students' difficulties. The next researcher should add more variables to define user behavior. This research result is $50 \%$ affected by non-tested variables. The next researcher should use potential respondents 
using application intensively in their work. The next research should study on accountancy software.

Vol. 1 No. 1, January - April 2016 


\section{References}

Ari, Dessanti Putri Sekti. (2013). Pengaruh Technology Acceptance Model dan Pengembangannya Dalam Perilaku Menggunakan Core Banking System. Jurnal Keuangan dan Perbankan, 17 (2). 267-278.

Arifin, M. R dan Astuti, S.P. (2012) . Sistem Keuangan \& Perbankan Syariah. Buku Praktikum Sistem Keuangan Perbankan Syariah.

Anggadini, S.D. (2014). Perlunya Akuntansi Syariah di Lembaga Bisnis (Keuangan) Syariah. Jurnal Ilmiah UNIKOM, 8 (2).

Budianto. (2009). Evaluasi Kesuksesan Sistem Informasi Dengan Pendekatan Model Delone Dan McLean Studi Kasus Implementasi Billing System di RSUD Kab. Sragen. Tesis Tidak Diterbitkan, Program Studi Magister Akuntansi Fakultas Ekonomi Universitas Sebelas Maret Surakarta, Surakarta.

Darmawan, D dan Fauzi. (2013). Metodologi Penelitian Kuantitatif. Bandung: PT Remaja Rosdakarya.

Davis, F.D. (1993). User Acceptance Of Information Technology : System Characteristics, User Perception And Behavioral Impact. Int. J. ManMachine Studies 38, 475-478. . (1989). Perceived Usefulness, Perceived Ease Of Use, And User Acceptance Of Information Technology. MIS Quartery. 13 (3), 319340.

Ghozali, I. (2006). Structural Equation Modeling Metode Alternatif Dengan Partial Least Square (PLS). Semarang: Badan Penerbit Universitas Diponegoro.

Hamzah, A. (2009). Evaluasi Kesesuaian Model Keperilakuan Dalam 
Penggunaan Teknology Sistem Informasi Di Indonesia. Seminar Nasional Aplikasi Teknologi Informasi 2009 (SNATI 2009) Yogyakarta 20 Juni 2009.

Handayani, T., Kusrini, dan Sunyoto, A. (2013). Analisis Penerimaan Sistem Informasi Akademik Berbasis Web Menggunakan Technology Acceptance Model (TAM) Dan Usability Studi Kasus Pada STTNAS Yogyakarta. Jurnal.Vol V, No.1, Mei 2013.

Harahap, S.S. (2001). Akuntansi Islam. Jakarta: Bumi Aksara.

Hartono, J. (2013). Sistem Teknologi Informasi Bisnis. Jakarta:Salemba Empat.

IAI. (2006). ED PSAK Penyajian Laporan Keuangan Syariah. Indriantoro, N dan Supomo, B. (2012). Metode Penelitian Bisnis. Yogyakarta: BPFE.

Jogiyanto, H.M. (2007). Sistem Informasi Keprilakuan. Yogyakarta: CV Andi Offset. . (2009). Konsep Dan Aplikasi PLS (Partial Least Square) Untuk Penelitian Empiris. Yogyakarta: BPFE-Yogyakarta.

Kartikahadi, H., Sinega, R.U., Syamsul, M., dan Siregar, S.V. (2012). Akuntansi Keuangan Berdasarkan SAK Berbasis IFRS. Jakarta: Salemba Empat.

Kartika. (2013). Technology Acceptance Model: Menguji Keefektivan

Penerimaan Sistem Informasi Terpadu (SISTER) di Lingkungan Universitas Jember. Jurnal.Jurusan Akuntansi Fakultas Ekonomi Universitas Jember.

Kusumo, H.C. (2010). Analisis Penerimaan Mobile Banking (M-Banking) Dengan Kerumitan (complexity) Sebagai Variabel Eksternal Dengan Menggunakan Pendekatan Technology Acceptance Model (TAM). 
Skripsi pada Jurusan Akuntansi Fakultas Ekonomi Universitas Sebelas Maret Surakarta, Surakarta.

Makbuloh, D. (2012). Pendidikan Agama Islam Arah Baru Pengembangan Ilmu Dan Kepriadian Di Perguruan Tinggi. Jakarta: PT Raja Grafindo Persada.

Muhammad. (2000). Prinsip-Prinsip Akuntansi Dalam Alqur'an. Yogyakarta: UII Press.

Nasution, M.E., Setyo, B., Huda, N., Mufraeni, M.A., dan Utama, B.S. (2007). Pengenalan EksklusifEkonomi Islam. Jakarta: Kencana Perdana Media Group.

Noor, J. (2011). Metodologi Penelitian. Jakarta: Kencana Prenada Media Group.

Nurhayati, S dan Wasilah. (2011). Akuntansi Syariah di Indonesia. Jakarta: Salemba Empat.

Retnoningrum, dan Jalil, A. (2013). Analisis Technology Acceptance Model pada Koperasi dan UKM Menggunakan Sistem Online dengan Budaya sebagai Variabel Moderator. Jurnal, Politeknik Pratama Mulia Surakarta.

Sangadji, E.M dan Sopiah. (2010). Metodologi Penelitian Pendekatan Praktis Dalam Penelitian Empiris. Yogyakarta:Andi.

Sanusi, A. (2011). Metodologi Penelitian Bisnis. Jakarta: Salemba Empat.

Sekaran, U. (2006). Research Methods for Business. Jakarta: Salemba Empat.

Santoso, B. (2010). Pengaruh Preceived Usefulness, Ease Of Use dan Perceived Enjoyment Terhadap Penerimaan Teknologi Informasi Studi Empiris Di Kab. Sragen. Jurnal Studi Akuntansi Indonesia, Fakultas Ekonomi Universitas Sebelas Maret Surakarta, Surakarta.

Shalihin, M dan Ratmono, D. (2013). Analisis SEM-PLS dengan WarpPLS 
3.0. Yogyakarta: CV. Andi Offset.

Singgih, E.M Dan Bawono, I.R. (2010). Pengaruh Independensi,

Pengalaman, Due Profesional Care, Dan Akuntabilitas Terhadap Kualitas Audit Studi Pada Auditor Pada KAP "BIG FOUR" Di Indonesia. Jurnal, SNA XIII Purwokerto 2010.

Sumiyanto, A. (2008). BMT Menuju Koperasi Modern. Yogyakarta: ISES Publishing.

Supardi. (2005). Metodologi Penelitian Ekonomi Dan Bisnis. Yogyakarta:UII Press.

Suarta, I.M Dan Sudiadyani, I.O. (2014). Studi Faktor Penentu Penerimaan dan Penggunaan Sistem Informasi Akuntansi Pada Lembaga Perkreditan Desa. 45 Jurnal Of Information System, 10 (1).

Sugiyono. (2012). Meotode Penelitian Kombinasi. Bandung: Alfabeta.

Venkatesh, V., Moris, M.G., Davis, G.B dan Davis, F.D. (2003). User Acceptance Of Information Technology: Toward a Unified View. MIS Quertery, 27 (3), 425-478.

Wijayanti, R. (2009). Analisis Technology Acceptance Model (TAM) Terhadap Faktor-Faktor Yang Mempengaruhi Penerimaan Nasabah Terhadap Layanan Internet Banking Studi Empiris Terhadap Nasabah Bank DiDepok. Skripsi pada Jurusan Akuntansi Fakultas Ekonomi Universitas Gunadharma.

Yusman. (2013). Analisis Sistem Informasi Keuangan Daerah Dengan Menggunakan Technology Acceptance Model Padad Pemerintah Daerah Kab. Sinjay. Skripsi pada Jurusan Akuntansi Fakultas Ekonomi Dan Bisnis Universitas Hassanudin, Makassar. 


\section{Online}

Angelia, L. (2015). Peranan Sistem Informasi Akuntansi Pada Perbankan Syariah. Accessed from https://linkaangelia.wordpress.com/19 Agustus 2015

Anything. (2015). Sistem Informasi Perbankan. Diakses pada 19 Agustus 2015 di https://infoseputarperbankan.wordpress.com.

Fathinah, F.K dan Baridwan, Z. (2012). Determinan Minat Individu dan Pengaruhnya Terhadap Perilaku Penggunaan Sistem Informasi Berbasis Teknologi di Bank Syariah. Accessed from http://datamatkul. blogspot.co.id/2013/06/determinana-minat-individu-dan.html.

Hastuti, K. (2014). Sistem Informasi Keuangan. Accessed from http:// khastuti.blogspot.com/19 Agustus 2015

Widiatmika, I.M.A.A dan Sensuse, I. (2014). Pengembangan Model Penerimaan Teknologi Internet Oleh Pelajar Dengan Menggunakan Konsep Technology Accptance Model (TAM). Jurnal Sistem Informasi MTI-UI, Vol.4, No.2. Retrieved from http://search.yahoo. com,06/09/2014. 